Objective The aim of study is to investigate the temporal trend and spatial pattern of mortality for malignant pleural mesothelioma in Taiwan during recent decades.

Method The standardized rates of mortality for MPM (ICD-9: 163, ICD-10: C45.0 \& C45.9) in Taiwan were computed at national and regional levels during 1975-2019; the sex ratios of male to female deaths for MPM were also computed at regional level.

Result The trend of national mortality for MPM in Taiwan is still increasing in twenty to thirty years after the asbestos control, especially among male population; the pattern of regional mortality for MPM reflects the distribution of the asbestosrelated industrial settlements in the country.

Conclusion In adequate response to the epidemic of asbestosrelated diseases, it is necessary to implement a national comprehensive program for the surveillance, diagnosis, and treatment healthcare to protect workers and community people.

\section{0-284 ENHANCING OCCUPATIONAL DISEASE SURVEILLANCE USING A JOB EXPOSURE MATRIX}

${ }^{1}$ Ashley Lau, Tracy Kirkham, Jill MacLeod, Paul Demers. 'Ontario Health, Canada

\subsection{6/OEM-2021-EPI.42}

Introduction Surveillance is useful for identifying occupations and industries at high risk of disease. However, less is known about workers' risks from exposure to specific occupational hazards.

Objectives Apply a job-exposure matrix to occupational disease risk estimates to enhance surveillance efforts.

Methods The Occupational Disease Surveillance System (ODSS) uses Cox proportional hazards models to derive health risks for $30+$ health outcomes. The Canadian jobexposure matrix (CANJEM) is a general population JEM that provides exposure data on over 250 hazards by occupation and industry. Exposure data for prevalence, intensity (nonexposed, low, medium, high), and duration of exposure (proportion of workweek exposed) were extracted. Hazard ratios (HRs) for silicosis and lung cancer from the ODSS were linked to silica exposure metrics from CANJEM by occupation (4-digit Canadian Classification and Dictionary of Occupations) and industry (3-digit 1970 Standard Industrial Classification).

Results There were 163 occupations and 141 industries exposed to silica according to CANJEM. Among occupations with $>5$ cases and $\geq 50 \%$ prevalence, the mean HRs were 18.33 (range: 16.5-19.7) and $1.25(0.77-1.87)$ for silicosis and lung cancer respectively. Similar results were seen with occupations with $\geq$ medium intensity, and $\geq 25 \%$ duration of exposure. Occupations with both $\geq 50 \%$ prevalence and $\geq$ medium intensity had mean HRs 18.33 (16.5-19.7) and 1.44 (1.16-1.87) for silicosis and lung cancer respectively. Industry results had similar distributions for lung cancer HRs, and lower mean HRs for silicosis (at $\geq 50 \%$ prevalence HR: 8.57, range: $1.48-19.3$ ).

Conclusion Using silica, we demonstrated that incorporating exposure information can help to identify and quantify workers' exposure risk. Incorporating multiple exposure metrics improved detection of high-risk groups although is less sensitive. Understanding the role of exposure on health risk may allow prevention strategies to be implemented more pragmatically.

\section{Exposure Assessment}

\section{0-94 DEVELOPMENT OF TASK-SPECIFIC ENDOTOXIN CONCENTRATIONS FOR AGRICULTURAL ACTIVITIES USING META-REGRESSION OF PUBLISHED DATA}

'Shuai Xie, Jean-Francois Sauve, Sarah Locke, Laura Beane Freeman, Melissa Friesen, Jonathan Hofmann, Pabitra Josse, Susan Viet, Felicia Hung. ' U.S. National Cancer Institute, United States

\subsection{6/OEM-2021-EPI.43}

Introduction Endotoxin has been hypothesized to partly account for the observed deficit of lung cancer in farmers; however, most epidemiologic studies have relied on surrogate metrics, such as number of animals.

Objective To obtain task-specific estimates of endotoxin exposure for agricultural tasks using meta-regression models of published data.

Methods We extracted the geometric means (GM, in EU/m3) and geometric standard deviations (GSD) of endotoxin measures for various farming activities from 43 published studies (1989-2018). We linked each measure to an activity within the questionnaire used in the Biomarkers of Exposure and Effect in Agriculture Study. When necessary, we calculated the GM and GSD from other available measures. We used mixed-effects meta-regression models with the weighted log-transformed GM as the dependent measure, task as the independent measure, and summary statistic identifier as a random effect to account for between-study heterogeneity. We grouped the tasks into three categories (crop, animal, stored seed/grain), and analyzed each category separately. We conducted sensitivity analyses by restricting data to only North America, only task-based, and only inhalable fraction.

Results We extracted 30 crop, 90 livestock, and 10 seed/grainrelated summary statistics. Among animal tasks, most had predicted GMs above $1000 \mathrm{EU} / \mathrm{m} 3$, including work in poultry confinement $(\mathrm{GM}=1470 \mathrm{EU} / \mathrm{m} 3)$, cleaning poultry confinements (1470), grinding feed (1410), work in swine confinement (1310), cleaning swine confinement (1270), feeding swine (1070), and veterinarian services (1030). Among crop tasks, predicted GMs were below $100 \mathrm{EU} / \mathrm{m} 3$, including harvesting corn/grains (30), hauling grain (60), and mowing (80). For stored seed/grain tasks, the predicted GM of cleaning grain bins was $1130 \mathrm{EU} / \mathrm{m} 3$ and other work with stored grains/seeds was $230 \mathrm{EU} / \mathrm{m} 3$.

Conclusion Our characterization of task-specific endotoxin concentrations can be used in conjunction with questionnaire responses on agricultural activities, including task duration, to improve future endotoxin assessments in epidemiologic studies.

\section{0-122 DETERMINANTS OF TASK-BASED EXPOSURES TO ALPHA-DIKETONES IN COFFEE ROASTING AND PACKAGING FACILITIES}

${ }^{1}$ Brie Blackley, Caroline Groth, Jean Cox-Ganser, Alyson Fortner, Ryan LeBouf, Xiaoming Liang, Abbas Virji. 'National Institute for Occupational Safety and Health, United States

\subsection{6/OEM-2021-EPI.44}

Introduction Coffee production is a global industry and is estimated to increase by 15.4 million $\mathrm{lbs}$ in 2021. Coffee 
production workers can be exposed to inhalational hazards such as diacetyl and 2,3-pentanedione, which are associated with the development of occupational respiratory disease, including obliterative bronchiolitis, a rare and irreversible lung disease.

Objective Identify determinants contributing to task-based exposures to alpha-diketones, specifically diacetyl and 2,3-pentanedione, at 17 U.S. coffee roasting and packaging facilities.

Methods We collected 606 personal task samples including roasting $(n=189)$, grinding $(n=74)$, packaging $(n=203)$, quality control $(n=44)$, flavoring $(n=15)$, and miscellaneous production/café $(n=81)$. Samples were collected and analyzed according to the modified OSHA Method 1013/1016. Information on sample-level and process-level factors relating to production scale, sources of alpha-diketones, and engineering controls was collected during surveys. Bayesian mixed-effect regression models accounting for censored data were fit for overall data and specific tasks including roasting, grinding, packaging, quality control, and flavoring. Significant determinants were used in multiple regression models using variable selection or model averaging Bayesian methods.

Results Task-based sample durations ranged from 2-86 minutes. Total number of alpha-diketone sources, sum of all open storage sources, average roasted coffee production per day, average percent of production as ground coffee, flavoring during survey, and number of grinders all resulted in increased exposures for at least one task category. General exhaust ventilation (GEV) with natural ventilation, supply air turned on, and GEV turned on resulted in lowered exposures for at least one task category.

Conclusions GEV and supply air turned on and natural ventilation were identified in most models as determinants which reduced exposures. Open storage of roasted coffee and flavoring during survey were significant in most models contributing to higher task exposures and can be targeted for exposure mitigation to reduce short-term and full-shift exposures and minimize risks for respiratory disease.

\section{0-137 ASSESSMENT OF MULTIPLE EXPOSURES TO CHEMICAL AGENTS IN FRENCH WORKPLACES: FINDINGS FROM TWO EXPOSURE DATABASES}

${ }^{1}$ Jean-Francois Sauve, Andrea Emili, Gautier Mater. ${ }^{1}$ Institut national de recherche et de sécurité (INRS), France

\subsection{6/OEM-2021-EPI.45}

Background National surveys of the French working population estimate that approximately $15 \%$ of all workers are exposed to at least three different chemical agents at work. However, the most prevalent coexposure situations and their associated health risks remain relatively understudied.

Objective To characterize occupational coexposure situations in France using available data from two occupational exposure databases.

Methods We extracted personal measurement data from the Colchic and Scola databases for the period 2010-2019. We selected 118 chemical agents that had $\geq 100$ measurements with detected concentrations, including 31 carcinogens (IARC groups 1, 2A, and 2B). We grouped measurements by work situation (WS, combination of sector, occupation, task, and year). We characterized the mixtures across WS using frequent itemset mining methods.

Results We retained 275,213 samples from 32,670 WS, encompassing 4,692 unique mixtures. Workers in thirty-two percent of all WS were exposed to $\geq 2$ agents (median 3 agents/WS, interquartile interval 2-5) and $13 \%$ of all WS contained $\geq 2$ carcinogens (median 2 carcinogens/WS, maximum 14). The most frequent coexposures across all agents were ethylbenzene-xylene (1,550 WS), quartz-cristobalite (1,417 WS), and toluene-xylene (1,305 WS). Prevalent combinations of carcinogens also included hexavalent chromiumlead (368 WS) and benzene-ethylbenzene (314 WS). Agents with the lowest proportions of coexposure were wood dust (6\% of WS exposed to at least one other agent) and asbestos $(8 \%)$. Tasks with the highest proportions of coexposure to carcinogens include electric arc welding (37\% of WS with coexposure), polymerization and distillation (34\%), construction drilling and excavating (34\%), and water collection and treatment $(32 \%)$.

Conclusion Coexposure to multiple chemical agents, including carcinogens, was highly prevalent in the databases, and should be taken into account when assessing exposure risks in the workplace. However, these databases do not necessarily represent a random sample of the working population, thereby limiting the generalizability of our findings.

\section{0-156 PESTICIDE EXPOSURE IN FRUIT GROWERS: COMPARISON OF LEVELS AND DETERMINANTS FROM THE CANEPA FIELD STUDY AND FROM THE 'AGRICULTURAL OPERATOR EXPOSURE MODEL' (AOEM) PREDICTIONS}

${ }^{1}$ Morgane Bresson, Mathilde Bureau, Jeremie Le Goff, Yannick Lecluse, Elsa Robelot, Isabelle Baldi, Pierre Lebailly. 'INSERM / Université Caen, France

\subsection{6/OEM-2021-EPI.46}

Introduction Pesticide exposures increase the risk of chronic diseases in farmers. Knowledge of exposure levels are needed for epidemiological studies and for regulation. In pesticide registration process, operator's exposure is predicted by the AOEM, set up in 2014 by the European Food Safety Authority, based on thirty studies conducted by the pesticide industry. To date, we are not aware of any field study comparing predicted data with those measured under real working conditions.

Objective We aimed to compare operators' exposures during treatment days in apple-growing under non-controlled conditions of work and values predicted by AOEM.

Methods Thirty apple growers from the French CANEPA study, were observed applying two fungicides (captan/dithianon) in 2016-2017. Dermal exposure was measured by body patches and cotton gloves. Detailed parameters about the farm, operator, application day, spraying equipment and personal protective equipment (PPE) used were recorded. For each observation, the corresponding exposure was calculated by the AOEM, using these parameters. The relationship between measured and calculated exposures was studied by linear regression.

Results Significant linear correlation was observed between calculated and measured daily exposures. Overall, the model 